\title{
1 A WHOLE-BRAIN 3D MYELOARCHITECTONIC ATLAS: MAPPING THE 2 VOGT-VOGT LEGACY TO THE CORTICAL SURFACE
}

3 Niels A. Foit MD ${ }^{1,2}$, Seles Yung ${ }^{1}$, Hyo Min Lee ${ }^{1}$, Andrea Bernasconi MD ${ }^{1}$, Neda Bernasconi

$4 \mathrm{MD} \mathrm{PhD}^{1^{*}}$, Seok-Jun Hong $\mathrm{PhD}^{1,3,4,5^{*}}$

$5{ }^{1}$ Neuroimaging of Epilepsy Laboratory, McConnell Brain Imaging Center, Montreal Neurological Institute, Montreal, QC,

6 Canada; ${ }^{2}$ Department of Neurosurgery, Medical Center - University of Freiburg, Freiburg, Germany; ${ }^{3}$ Center for Neuroscience

7 Imaging Research, Institute for Basic Science, Suwon, Korea; ${ }^{4}$ Department of Biomedical Engineering, Sungkyunkwan

8 University, Suwon, Korea; ${ }^{5}$ Center for the Developing Brain, Child Mind Institute, NY, USA

\section{Short title-Myeloarchitectonic MRI atlas}

14175 words in abstract / main text

163 color Figures; 3 tables

\section{Classifications (1minor / 1 major): Biological Sciences, Neuroscience}

18 Key words: Myeloarchitecture, MNI space, intracortical depth profiling, FreeSurfer

*) These authors share senior authorship of the manuscript.

\section{CORRESPONDING AUTHOR}

24 Seok-Jun Hong, PhD

25 Center for Neuroscience Imaging Research

26 Department of Biomedical Engineering

27 Sungkyunkwan University, Suwon, 16419, Korea

29 Center for the Developing Brain

30 Child Mind Institute, NY, USA

31 hongseokjun@skku.edu 


\section{FUNDING SOURCES}

36 This project was funded by CIHR MOP-57840 to AB and CIHR MOP-123520 to NB,

37 Natural Sciences and research Council (NSERC; Discovery-243141 to AB and 24779

38 to NB), Epilepsy Canada Jay and Aiden Barker grant (247394 to AB), Canada First

39 Research Excellence Fund (HBHL-1a-5a-06 to NB) and the German Research

40 Foundation (DFG, FO996/1-1 to NAF), the Brain \& Behavior Research Foundation

41 (NARSAD Young Investigator Award \#28436) and the Institute for Basic Science

42 (IBS-R15-D1 to SJH). The funders had no role in the design of the study, collection,

43 analyses, or interpretation of data, writing of the manuscript or the decision to publish

44 the results.

\section{CRediT author roles}

- Niels A. Foit: data curation, methodology, formal analysis, interpretation of data,

48 manuscript writing and editing.

49 - Seles Yung: structure segmentation, formal analysis

50 - Hyo-Min Lee: manuscript reviewing and editing

51 - Andrea Bernasconi: supervision, methodology, interpretation of data, manuscript

52 reviewing and editing.

53 - Neda Bernasconi: supervision, methodology, interpretation of data, manuscript $54 \quad$ reviewing and editing

55 - Seok-Jun Hong: data curation, supervision, methodology, formal analysis, 56 manuscript writing, reviewing and editing

\section{Declaration of interests}

59 The authors declare that there is no conflict of interest related to this manuscript. 


\section{Abstract}

62 Building precise and detailed parcellations of anatomically and functionally distinct

63 brain areas has been a major focus in Neuroscience. Pioneer anatomists parcellated

64 the cortical manifold based on extensive histological studies of post-mortem brain,

65 harnessing local variations in cortical cyto- and myeloarchitecture to define areal

66 boundaries. Compared to the cytoarchitectonic field, where multiple neuroimaging

67 studies have recently translated this old legacy data into useful analytical resources,

68 myeloarchitectonics, which parcellate the cortex based on the organization of

69 myelinated fibers, has received less attention. Here, we present the neocortical

70 surface-based myeloarchitectonic atlas based on the histology-derived maps of the

71 Vogt-Vogt school and its 2D translation by Nieuwenhuys. In addition to a

72 myeloarchitectonic parcellation, our package includes intracortical laminar profiles of

73 myelin content based on Vogt-Vogt-Hopf original publications. Histology-derived

74 myelin density mapped on our atlas demonstrate close overlap with in vivo

75 quantitative MRI markers for myelin and relates to cytoarchitectural features.

76 Complementing the existing battery of approaches for digital cartography, the whole-

77 brain myeloarchitectonic atlas offers an opportunity to validate imaging surrogate

78 markers of myelin in both health and disease. 


\section{Highlights (will go as a separate file)}

80 - Our myeloarchitectonic atlas builds on extensive meta-analyses-derived and 81 ground-truth histological data.

82

83 - Our atlas provides qualitative and quantitative 3D information on cortical 84 myelin architecture.

85

86 - MRI surrogate markers of myelin demonstrate close overlap with histological 87 cortical parcellations, supporting biological validity of non-invasive metrics.

- This atlas can be seamlessly integrated into widely used neuroimaging analysis software to inform studies in health and disease. 


\section{$91 \quad$ Introduction}

92 Obtaining precise and detailed parcellations of anatomically and functionally distinct

93 brain areas has been the focus of Neuroscience research for over a century (Zilles et

94 al., 2015; Zilles and Amunts, 2010). Among neuroanatomists of the early $20^{\text {th }}$ century,

95 Brodmann, Vogt and Vogt, and von Economo and Koskinas ardently worked towards

96 generating highly detailed histological maps of the human cortex based on post-

97 mortem data (Triarhou, 2007; Zilles and Amunts, 2010). To parcellate the neocortex,

98 they relied on cytoarchitectonics, characterizing size, shape and distribution of cell

99 bodies across cortical layers (Amunts et al., 2005; Smith, 1927; Triarhou, 2007), and

100 myeloarchitectonics, which studies layering, arrangement, packing and density of

101 myelinated fibers and bundles (Batsch, 1955; Hopf, 1968; Nieuwenhuys, 2013;

102 Strasburger, 1937; Vogt and Vogt, 1919). These histology-derived maps have set the

103 basis for MRI-derived in vivo parcellations of cortical boundaries (Huntenburg et al.,

104 2017; Mendes et al., 2019; Nieuwenhuys and Broere, 2020). Nevertheless, knowledge

105 on cytoarchitectonics today remains heavily influenced by the classic work of

106 Brodmann (Brodmann, 1907; Zilles and Amunts, 2010) and many contemporary

107 neuroimaging toolkits contain modified versions of Brodmann's seminal map

108 (Eickhoff et al., 2005; Talairach et al., 1993).

109 Compared to the cytoarchitectonic field, myeloarchitectonics has received less

110 attention. This may be due to the paucity of histology-based myeloarchitectonic data

111 to establish the biological substrates of several MRI markers for myelin

112 characteristics (Lazari and Lipp, 2021; van der Weijden et al., 2020), such as

113 quantitative T1 mapping (Lutti et al., 2014; Marques et al., 2017) and neurite

114 orientation and dispersion density imaging (Zhang et al., 2012). Recently,

115 Nieuwenhuys and co-workers (Nieuwenhuys and Broere, 2017) mapped Vogts 
116 myeloarchitectonic atlases to a non-digital, 2D representation of the Montreal

117 Neurological Institute (MNI) Colin27 brain template, by means of manual translations

118 from paper-embedded figures. Harnessing the data generated by Hopf, they further

119 integrated area-specific myelin fiber density estimates into their myeloarchitectonic

120 map (Hopf, 1968, 1957, 1956, 1955; Nieuwenhuys and Broere, 2017).

121 While 2D maps render the knowledge from historical postmortem data more

122 accessible, they cannot be used in quantitative neuroimaging analyses, which usually

123 require 3D stereotaxic volumetric data representation or cortical surface formats. In an

124 effort to address this gap, we built a 3D myeloarchitectonic atlas (MYATLAS) in

125 common space, translating the Nieuwenhuys' boundaries of the Vogt-Vogt atlas to the

126 Colin27 brain template and standard cortical surfaces. Besides providing a ready-to-

127 use myeloarchitectonic parcellation, we generated intracortical laminar profiles of

128 myelin content from the photometric data gathered from Vogt-Hopf publications. To

129 validate our atlas, we quantified the similarity between the histology-based myelin

130 density and profiles from in vivo MRI markers obtained from MP2RAGE-derived

131 qT1 mapping $(21,34,35)$. Moreover, we integrated cytoarchitectonic features

132 (Scholtens et al., 2018). Finally, to facilitate integration into existing image

133 processing pipelines, the proposed myeloarchitectonic atlas together with source

134 codes are made publicly available.

\section{Materials and Methods}

137 After a short review of prior work, sections below detail the methodology used for the

138 creation of our 3D myeloarchitectonic atlas (MYATLAS) and necessary processing

139 steps to obtain cortical depth profiles from Vogt-Vogt myeloarchitectonic

140 parcellations, as well as cross-modal correlations between myelo- and 
141 cytoarchitectural features and in vivo qT1 mapping. We further provide instructions

142 for registering the MYATLAS to individual MRI data.

\section{Summary of prior work}

144 Nieuwenhuys and co-workers recently performed a meta-analysis on potential

145 usability of the historical myeloarchitectural data aggregated from Vogts' papers (see

146 Nieuwenhuys and Broere, 2017 for details). The original cortical division by the

147 Vogts school consisted of a total of 180 myeloarchitectonic cortical fields (64 frontal,

14830 parietal, 63 temporal, 17 occipital, and 6 insular areas). Their associates Hopf and

149 Vitzthum further refined this work by subdividing the parietal and occipital lobes into

150 multiple sub-areas (Hopf, 1957, 1956, 1955), resulting in 214 regions (64 frontal, 60

151 parietal, 63 temporal, 21 occipital, and 6 insular). Nieuwenhuys et al. applied semi-

152 automatic topological translations and boundary averaging across 17 different views

153 of this analog dataset to project myeloarchitectonic parcellations onto the MNI-

154 Colin27 single subject brain in a non-digital figure format (Figure 1).

155 In addition to boundaries, the Vogts investigated intracortical penetration patterns of

156 tangential and radial fiber bundles across cortical laminae (Vogt and Vogt, 1919).

157 They defined major categories of myeloarchitectonic profiles based on two criteria

158 (Figure 2A). The first criterion related to the presence of transverse, densely

159 myelinated cortical layers (the bands of Baillarger). Accordingly, cortical specimens

160 can be classified into 4 categories: bistriate (two horizontal myelin-rich bands),

161 unistriate and unitostriate (both indicating only one visible band; for the former, a

162 single cortical layer is covered, whereas for the latter multiple layers are covered by

163 the band), and astriate (no striation). Notably, each category has a subtype, depending

164 on the demarcation of a band boundary (propebistriate: barely recognizable bands of

165 Baillarger; propeunistriate: ill-defined border of inner stripe; propeastriate: slight 
166 decrease of density in 5a/6a). The second criterion relates to the intrusion depth of the

167 radiate bundles across cortical laminae, further classifying the cortex into euradiate

168 (bundles reaching upper border of layer 3b), infraradiate (reaching upper border of

169 layer 5b), and supraradiate (extending into layers 1-2).

170 Following this work, Hopf (Hopf, 1968, 1957, 1956, 1955) further advanced these

171 myeloarchitectonic parcellation by systematically recording frontal, parietal and

172 temporal lobe myelin contents using analog photodensitometry. Notably, each region

173 has distinct light absorption curves, or cortical depth profiles. This data was recently

174 digitized by Nieuwenhuys (Nieuwenhuys and Broere, 2017), who introduced mean

175 grey levels (MGL), i.e., digital quantifications of myelin fiber density per cortical

176 area. Ranging from 0 to 255 , lower values indicate densely myelinated areas, while

177 high MGL are found in lightly myelinated areas (Edwards et al., 2018; Nieuwenhuys

178 and Broere, 2017). Since MGL are only available for the frontal, parietal and temporal

179 lobes, remaining areas were assigned 255 as a default value. Since Hopf based his

180 work exclusively on the right hemisphere, MGL were available only for the right

181 hemisphere in Nieuwenhuys' work (Nieuwenhuys and Broere, 2017).

$\underline{\text { MRI processing }}$

184 We created a stereotaxic average of the individual Colin27 brain MRIs, comprising 27

185 T1 weighted scans with $1 \mathrm{~mm}$ isotropic voxel resolution (Holmes et al., 1998). We

186 then extracted 3D cortical surface models from this template using FreeSurfer (Fischl,

187 2012). Briefly, processing steps included gradient non-uniformity correction (Jovicich

188 et al., 2006), registration to MNI stereotaxic space, intensity normalization, skull

189 stripping, and segmentation into tissue classes (Fischl et al., 2004). Gray-white and

190 gray-CSF interface models were generated through triangular surface tessellation 
191 yielding 163,842 vertices (Dale et al., 1999), followed by topology correction,

192 inflation, and spherical registration to fsaverage (Fischl et al., 2001).

\section{2D-to-3D translation of myeloarchitectural parcellations.}

194 As per previous procedures (Pijnenburg et al., 2021; Scholtens et al., 2015), the 195 original Nieuwenhuys' illustration (Nieuwenhuys et al., 2015) was split into eight 196 view planes (lateral, medial, superior, inferior, orbitofrontal, supratemporal, parietal 197 opercular, insular). Carefully cross-referencing different views, a single rater (SY) 198 labeled each parcellation by comparing geometric landmarks between Nieuwenhuys' 199 illustrations and the convexity of the 3D Colin27 brain surface. Labelling was 200 performed with "tksurfer" (surfer.nmr.mgh.harvard.edu/fswiki/TkSurfer). Notably, 201 identifiable sulci on the original 2D Colin27 map such as the central and superior 202 temporal sulcus served as systematic landmarks. To optimize anatomical matching of 203 area boundaries, we further relied on the main sulcal patterns surrounding each region 204 to be labeled. A second rater (SJH) evaluated accuracy of each label. For ambiguous 205 areas, i.e., either a mismatch across view planes, or between 2D illustrations and 3D 206 cortical surface, an inter-rater consensus on boundaries was reached to minimize

207 discrepancies by carefully reviewing the original publications and applying manual 208 corrections, if necessary (Supplementary Table 1).

209 An inherent limitation of Vogt-Vogt histological data is that all results were reported 210 on the convex pial surface, which does not reveal buried sulci. We thus labelled areas 211 located within these sulci on the white matter surface view on which they are clearly 212 visible and intra-sulcal boundaries can be easily delineated at their bottom. To 213 visualize areas hidden in the depths of the Sylvian fissure (i.e., insula, supratemporal 214 lobe, parietal operculum), invisible even on the white matter surface, we extracted 215 patches of their surfaces, delineated label boundaries and merged them back to the 
216 whole-brain data. Notably, some areas appeared multiple times across view planes,

217 preventing their segmentation into a single coherent label. This was addressed by

218 dividing areas into sub-regions; for example, for area 111 appearing differently in the

219 lateral and medial views, we divided it into 111-1, 111-m, 'l' and 'm' refer to medial

220 and lateral, respectively (see Supplementary Table 1). Finally, resulting labels were

221 numbered in accordance to Vogt's numeric convention and merged with color tables

222 to create a single Freesurfer annotation file (.annot), which contains a total of 214

223 parcellations (Figure 1C).

Quantitative cortical myelin content and intracortical depth profiling

226 MRI allows for in vivo quantification of myelin content of the cortical manifold

227 (Stüber et al., 2014; Waehnert et al., 2014). However, validation requires access to

228 histology, which has not been available in a digital format. We thus generated

229 histology-derived quantitative myelin data by recording the MGL index (the averaged

230 myelin fiber density) for each cortical field (Nieuwenhuys and Broere, 2017) and

231 created a ready-to-analyze look-up table in excel format for the use with the

232 MYATLAS (Supplementary Material 1, "Myeloarchitectural_table.xslx"). As the

233 MGL for the insula and occipital lobes (34 parcels) were unavailable, they were

234 omitted, totaling 187 values (64 frontal, 60 parietal and 63 temporal areas;

235 Supplementary Material 1). We then mapped the myelination density (MGL values)

236 onto the MYATLAS (Figure 1).

237 To extract myelin laminar depth profiles from histologically-stained

238 microphotographs (Batsch, 1955; Brockhaus, 1940; Hopf, 1957, 1956, 1955;

239 Strasburger, 1937; Vogt and Vogt, 1919), we screen-captured the histology photos

240 with a fixed format and size, and estimated the gray level intensity across cortical 
241 laminae as a surrogate of myelin density. The digitized histological figures were

242 normalized to make intensity values across photos comparable. Absolute gray values

243 were then extracted and plotted as a normalized depth profile across all cortical layers

244 (Figure 2B). Information on myeloarchitectonic features, such as fiber bundle types

245 and layer-specific density of each cortical stain, were also recorded (Supplementary

246 Material 1, Figure 2C).

248 Correlation between myeloarchitectonic features and in-vivo myelin proxy data

249 We cross-validated myeloarchitectonic features through associations with qT1 250 mapping (Edwards et al., 2018; Mancini et al., 2020; Weiskopf et al., 2015).

251 Compared to conventional weighted sequences, MP2RAGE-derived qT1 images are

252 inherently uniform, theoretically free of other imaging properties like proton density

253 or $\mathrm{T} 2 *$, and are acknowledged as directly relating to cortical myelin content (Marques

254 et al., 2017; Marques and Gruetter, 2013; van der Weijden et al., 2020). For qT1

255 sampling, we selected the 202 individuals from the Leipzig Study for Mind-Body-

256 Emotion Interactions (LEMON) dataset (Babayan et al., 2019). Details of LEMON

257 acquisition protocols and preprocessing steps have been described in detail (Mendes

258 et al., 2019).

259 To correlate the depth of profiles acquired from digitized histological data

260 microphotographs with in vivo intracortical qT1, we positioned 10 equivolume

261 surfaces between the inner and outer cortical interface using

262 equivolumetric_surfaces.py (https://github.com/kwagstyl/surface_tools.git). These

263 surfaces systematically sampled the axis perpendicular to the cortical ribbon, with

264 interpolation at each vertex (Hong et al., 2017, 2016).

265 Correlation of myelin content with von Economo-Koskinas cytoarchitectonic data 
266 To verify the neurobiological significance of MYATLAS, we correlated the MGL

267 with von Economo-Koskinas' cytoarchitectonic features of gyral dome thickness, 268 cellular density and cell size (Scholtens et al., 2018, 2015). Since gyral dome

269 thickness is reported as a range, region-specific averages were calculated. Cellular

270 densities were averaged across all cortical layers, whereas cell size was calculated

271 according to $\left[\mathrm{H}_{\text {mean }} \mathrm{x} \mathrm{W}_{\text {mean }}\right]$; with $\mathrm{H}_{\text {mean }}($ Height $)=\left[\mathrm{H}_{(\text {min-max })} / 2\right]$ and $\mathrm{W}_{\text {mean }}($ Width $)=$

$272\left[\mathrm{~W}_{(\min -\max )} / 2\right]$ per individual cortical layer and then averaged across all layers. To

273 allow for between-atlas correlation, we matched the boundary of parcels using a

274 winner-takes-all approach to assign each parcel of the von Economo-Koskinas' atlas 275 to our parcellation.

277 Use of the MYATLAS and associated features

278 All parcellations and MGL maps are available in two widely used formats (gifti and 279 nifti ['dlabel' for MGL and 'dscalar' for parcellation]) and two brain spaces (Colin27 280 and Conte69, both with 32k vertices). We further provide the original, manual parcel 281 translation file ('rh.vogt_vogt.annot') for use with FreeSurfer. Finally, to facilitate 282 implementation, we also provide Bash scripts which convert the original labels from 283 the MNI-Colin27 brain to single-subject space using FreeSurfer 'mri_label2label' 284 (mapping_colin27_labels_onto_individuals[_batch].sh). Finally, we generated a 285 flipped version of the left hemisphere atlas based on symmetric hemispheric 286 registration ('xhemi' command in FreeSurfer). 


\section{Results}

\section{$\underline{3 D \text { myeloarchitectonic atlas }}$}

290 The MGL patterns were similar between the MYATLAS and the Nieuwenhuys map

291 (Figure 1). Indeed, the lowest MGL values were found in highly myelinated primary

292 sensory areas (somatosensory areas 67, 69-71II of the postcentral gyrus; auditory 293 cortex, areas 145-157) and primary motor cortices (areas 39, 42, 43). Notably, similar

294 to Vogt's observation of continuous changes of architectural features, our 3D map 295 displayed gradually decreasing myelin content in areas distant from primary cortices.

296 Reflecting this pattern, higher-order areas (including areas 49-51 of the frontal pole) 297 and precuneus (areas 81-85) revealed higher MGL (i.e., less myelination), compared 298 to the rest of the brain. There were some noteworthy exceptions to this pattern of 299 hierarchy-dependent myelin profiles, previously recognized by Vogt, with densely 300 myelinated clusters, comprising the orbitofrontal (60 and 61), intraparietal $(86,87)$, 301 and posterolateral (169-172) and basal (173-177, 179-180) temporal areas. These

302 clusters have also been consistently identified both ex vivo and on structural MRI 303 (Glasser and Van Essen, 2011; Nieuwenhuys and Broere, 2017), all relating to visual 304 processing (Nieuwenhuys and Broere, 2017). Notably, areas 173,174 177,179-80

305 correspond to two newly discovered distinct cytoarchitectonic areas FG3 and FG4 of 306 the fusiform gyrus (Lorenz et al., 2017).

$308 \quad$ Intracortical depth profiling based on Vogt-Vogt classifications

309 Figure 2A-B illustrate 3D maps of fiber penetration patterns derived from Vogt-Hopf

310 studies stratified with respect to the presence of the bands of Baillarger and bundle

311 intrusion types (see Method 'prior work' for stratification details). Specifically, 312 within available areas, high-order cognitive regions showed either the unistriate 
313 (temporal and frontal areas) or bistriate (parietal) subtype. In contrast, the bundle

314 intrusion types were relatively homogeneous, with majority of the areas containing

315 the euradiate subtype. Figure 2C shows patterns of myelination density across lobes.

317 Correlation between surface-mapped MGL and in vivo myelin metrics

318 We found a positive correlation between our histology-derived MGL and in vivo qT1

319 values $\left(\mathrm{p}<5 \times 10^{-7}, \mathrm{r}=0.39\right)$, indicating close correspondence between these surrogate

320 metrics of myelin. Figure 3A illustrates the group-averaged qT1 map of 202 subjects

321 selected from the LEMON dataset (Babayan et al., 2019). The lowest qT1 values were

322 found in heavily myelinated primary cortices, with a decrease when moving towards

323 higher order areas. Notably, low qT1 values found in the posterolateral temporal lobe

324 likely indicate the dense myelination of the dark cluster described by Vogt

325 (Nieuwenhuys and Broere, 2017). Figure 3B illustrates the correspondence between

326 photodensitometric quantifications of cortical myelin content and in vivo qT1 depth

327 profiles.

\section{Correlation between surface-mapped MGL and cytoarchitectonic features}

330 Only cell size was found to correlate with myelin density as represented by MGL ( $\mathrm{r}=$

$331-0.27, \mathrm{p}<0.0001$; Figure 3C), where smaller cell sizes were associated with higher

332 myelin density. While gyral dome thickness trended towards a similar relationship (r

$333=-0.12, \mathrm{p}<0.09)$, cell density did not $(\mathrm{r}=0.03, \mathrm{p}>0.7)$. These findings are in line

334 with previous studies showing that axons of smaller sensory neurons are often

335 unmyelinated (Lee et al., 1986, p. 198). Notably, pyramidal axons of the central motor

336 cortex exhibit complex myelination patterns (Micheva et al., 2016), which differ

337 between cortical layers (Tomassy et al., 2014). Thus, it is conceivable that these 
338 complex interactions might not be captured by the limited resolution of a simplified

339 metric such as MGL.

$341 \quad$ Code and Data availability

342 The MYATLAS, lookup tables, source codes and the scripts for applying the atlas to

343 Colin27 and Conte69 brain templates as well as to individual brains

344 ("mapping_colin27_labels_onto_individuals_batch.sh") are available from this link

345 (https://bic.mni.mcgill.ca/ noel/noel-myelin). A README file together with detailed

346 descriptions of the downloadable files are available from the same web repository. All

347 imaging-derived files are in FreeSurfer MGH and NIFTI formats and can be viewed

348 with standard software (e.g., FreeView, FSLeyes or wb_view). The scripts used for

349 data processing are available from the authors upon request.

\section{Discussion}

352 Digital reconstructions of histological brain atlases constitute an important resource

353 for contemporary neuroimaging. Such reconstructions expand availability of

354 previously inaccessible, yet highly comprehensive, observations. Building upon the

355 Vogt legacy, we present the MYATLAS, a 3D myeloarchitectonic digital cartography

356 to assist neuroimaging mapping studies. To facilitate broad application, we provide

357 the atlas together with the codes and data files. Moreover, to mitigate inter-individual

358 variability, the parcellations and MGL maps are available both on a single- and a

359 multi-subject group templates in stereotaxic space. Integration of detailed,

360 quantitative data on cortical myelination will allow future neuroimaging research to 
361 assess their findings based on both myelin density and microstructure, enhancing

362 biological validity.

363 Variations in myelination relate to various aspects of neocortical structure and

364 function, including connectivity and hierarchical processing (Boshkovski et al., 2021;

365 Huntenburg et al., 2017; Royer et al., 2020). Moreover, disrupted myeloarchitectural

366 properties may reflect the pathological underpinning of neurological disorders (Nord

367 et al., 2019). Thus, classification of cortical myeloarchitectonic areas and patterns

368 through parcellation and MGL mapping has significant translational potential in both

369 health and disease. Future neuroimaging studies may leverage this information to

370 elucidate pathological whole-brain myeloarchitectural patterns, for instance in

371 multiple sclerosis (Rahmanzadeh et al., 2021) and epilepsy (de Curtis et al., 2021;

372 Drenthen et al., 2019).

373 Our atlas aggregates histology-derived myeloarchitectural information, depth-

374 dependent photometric density, as well as corresponding in vivo qT1 profiles, together

375 with myeloarchitectural subtypes categorized into laminar and depth intrusion

376 patterns of fiber bundles. Notably, the high congruence between histology-derived

377 quantifications of myelin density trough MGL and qT1 lends further biological

378 validity to this in vivo microarchitectural surrogate metric easily implementable in

379 clinical settings (Hogan, 2017; Waehnert et al., 2016). Notably, recent developments

380 in advanced imaging sequences, such as myelin water imaging (van der Weijden et

381 al., 2020) or high-field laminar fMRI (Trampel et al., 2019), allow for an increasingly

382 detailed study of cortical myelin contents. In this regard, our histology-validated

383 depth profiles can be harnessed as ground-truth data to validate future in vivo imaging

384 studies investigating myeloarchitecture (O'Muircheartaigh et al., 2019; Yuan et al., 385 2021). 
386 A few noteworthy points should be considered when applying MYATLAS to new

387 data. First, as this atlas is based on consensus evidence (i.e., cortical boundaries) from

388 several studies and does not incorporate information on inter-individual variability of

389 myeloarchitectural characteristics. This limitation may however be mitigated by

390 employing our atlas in conjunction with probabilistic cortical mapping approaches,

391 such as Julich Brain (Amunts et al., 2020). Additionally, our digital atlas does not

392 contain information on potential left-right asymmetries, since the original sources also

393 do not contain any lateralization information. Nevertheless, a recent quantitative MRI

394 study investigating myeloarchitectural metrics of the language system revealed

395 heterogenous lateralization patterns (Yuan et al., 2021): While inferior frontal areas

396 were left lateralized, the middle and superior temporal gyrus (Heschl's gyrus and

397 planum temporale) was found to be right lateralized. As such, future research should

398 therefore be directed at potential functional implications of myeloarchitectural

399 lateralization patterns in larger cohorts. Additionally, since measures of myelination

400 density (namely MGL and cortical depth profiles) are unavailable for the occipital

401 lobe and the insula, a whole-brain neuroimaging correlation remains somewhat

402 partial. However, due to their high congruence, this limitation could be resolved by

403 extrapolating MGL from qT1 in vivo data, preferably acquired at ultra-high magnetic

404 field strengths (Sengupta et al., 2018).

405 Future applications of our architectonic mapping framework may include correlations

406 between myeloarchitectonic density and myelin-related genes (Donkels et al., 2020;

407 Glasser et al., 2016). For instance, building on our procedures from MGL-qT1 cross-

408 correlation, it is now possible to relate myelin-related gene expression to

409 myeloarchitectonic features within individual cortical parcellations. Such efforts may 
bioRxiv preprint doi: https://doi.org/10.1101/2022.01.17.476369; this version posted January 20, 2022. The copyright holder for this preprint (which was not certified by peer review) is the author/funder. All rights reserved. No reuse allowed without permission.

410 provide further insights on the role of specific genes in health and disease

411 (Rahmanzadeh et al., 2021; Sprooten et al., 2019).

412 


\section{References}

414

415

416

417

418

419

420

421

422

423

424

425

426

427

428

429

430

431

432

433

434

435

436

437

438

439

440

441

442

443

444

445

446

447

448

449

450

451

452

453

454

455

456

457

458

459
Amunts K, Kedo O, Kindler M, Pieperhoff P, Mohlberg H, Shah NJ, Habel U, Schneider F, Zilles K. 2005. Cytoarchitectonic mapping of the human amygdala, hippocampal region and entorhinal cortex: intersubject variability and probability maps. Anat Embryol (Berl) 210:343-352. doi:10.1007/s00429005-0025-5

Amunts K, Mohlberg H, Bludau S, Zilles K. 2020. Julich-Brain: A 3D probabilistic atlas of the human brain's cytoarchitecture. Science 369:988-992. doi:10.1126/science.abb4588

Babayan A, Erbey M, Kumral D, Reinelt JD, Reiter AMF, Röbbig J, Schaare HL, Uhlig M, Anwander A, Bazin P-L, Horstmann A, Lampe L, Nikulin VV, Okon-Singer H, Preusser S, Pampel A, Rohr CS, Sacher J, Thöne-Otto A, Trapp S, Nierhaus T, Altmann D, Arelin K, Blöchl M, Bongartz E, Breig P, Cesnaite E, Chen S, Cozatl R, Czerwonatis S, Dambrauskaite G, Dreyer M, Enders J, Engelhardt M, Fischer MM, Forschack N, Golchert J, Golz L, Guran CA, Hedrich S, Hentschel N, Hoffmann DI, Huntenburg JM, Jost R, Kosatschek A, Kunzendorf S, Lammers H, Lauckner ME, Mahjoory K, Kanaan AS, Mendes N, Menger R, Morino E, Näthe K, Neubauer J, Noyan H, Oligschläger S, Panczyszyn-Trzewik P, Poehlchen D, Putzke N, Roski S, Schaller M-C, Schieferbein A, Schlaak B, Schmidt R, Gorgolewski KJ, Schmidt HM, Schrimpf A, Stasch S, Voss M, Wiedemann A, Margulies DS, Gaebler M, Villringer A. 2019. A mind-brain-body dataset of MRI, EEG, cognition, emotion, and peripheral physiology in young and old adults. Sci Data 6:180308. doi:10.1038/sdata.2018.308

Batsch E-G. 1955. Die myeloarchitektonische Untergliederung des Isocortex parietalis beim Menschen. J Hirnforsch 46.

Boshkovski T, Kocarev L, Cohen-Adad J, Mišić B, Lehéricy S, Stikov N, Mancini M. 2021. The R1-weighted connectome: complementing brain networks with a myelin-sensitive measure. Netw Neurosci 5:358-372. doi:10.1162/netn_a_00179

Brockhaus H. 1940. Die Cyto- und Myeloarchitektonik des Cortex claustralis und des Claustrum beim Menschen. J Fuer Psychol Neurol 49:100.

Brodmann K. 1907. Beiträge zur histologischen Lokalisation der Grosshirnrinde: Die Cortexgliederung des Menschen. VI. Verlag von Johann Ambrosius Barth.

Dale AM, Fischl B, Sereno MI. 1999. Cortical surface-based analysis. I. Segmentation and surface reconstruction. NeuroImage 9:179-194. doi:10.1006/nimg.1998.0395

de Curtis M, Garbelli R, Uva L. 2021. A hypothesis for the role of axon demyelination in seizure generation. Epilepsia 62:583-595. doi:10.1111/epi.16824

Donkels C, Peters M, Núñez MTF, Nakagawa JM, Kirsch M, Vlachos A, Scheiwe C, Schulze $\square$ Bonhage A, Prinz M, Beck J, Haas CA. 2020. Oligodendrocyte lineage and myelination are compromised in the gray matter of focal cortical dysplasia type IIa. Epilepsia doi:https://doi.org/10.1111/epi.16415

Drenthen GS, Wald ELAF, Backes WH, Hall MHJAD-V, Hendriksen JGM, Aldenkamp AP, Vermeulen RJ, Klinkenberg S, Jansen JFA. 2019. Lower 
myelin-water content of the frontal lobe in childhood absence epilepsy. Epilepsia 60:1689-1696. doi:https://doi.org/10.1111/epi.16280

Edwards LJ, Kirilina E, Mohammadi S, Weiskopf N. 2018. Microstructural imaging of human neocortex in vivo. NeuroImage, Microstructural Imaging 182:184206. doi:10.1016/j.neuroimage.2018.02.055

Eickhoff SB, Stephan KE, Mohlberg H, Grefkes C, Fink GR, Amunts K, Zilles K. 2005. A new SPM toolbox for combining probabilistic cytoarchitectonic maps and functional imaging data. NeuroImage 25:1325-1335. doi:10.1016/j.neuroimage.2004.12.034

Fischl B. 2012. FreeSurfer. NeuroImage 62:774-781. doi:10.1016/j.neuroimage.2012.01.021

Fischl B, Liu A, Dale AM. 2001. Automated manifold surgery: constructing geometrically accurate and topologically correct models of the human cerebral cortex. IEEE Med Imaging 20:70-80.

Fischl B, Salat DH, Kouwe AJW van der, Makris N, Ségonne F, Quinn BT, Dale AM. 2004. Sequence-independent segmentation of magnetic resonance images. NeuroImage 23:S69-S84. doi:DOI: 10.1016/j.neuroimage.2004.07.016

Glasser MF, Coalson TS, Robinson EC, Hacker CD, Harwell J, Yacoub E, Ugurbil K, Andersson J, Beckmann CF, Jenkinson M, Smith SM, Van Essen DC. 2016. A multi-modal parcellation of human cerebral cortex. Nature 536:171-178. doi:10.1038/nature18933

Glasser MF, Van Essen DC. 2011. Mapping human cortical areas in vivo based on myelin content as revealed by T1- and T2-weighted MRI. J Neurosci Off J Soc Neurosci 31:11597-11616. doi:10.1523/JNEUROSCI.2180-11.2011

Hogan RE. 2017. Quantitative Measurement of Longitudinal Relaxation Time (qT1) Mapping in TLE: A Marker for Intracortical Microstructure? Epilepsy Curr 17:358-360. doi:10.5698/1535-7597.17.6.358

Holmes CJ, Hoge R, Collins L, Woods R, Toga AW, Evans AC. 1998. Enhancement of MR Images Using Registration for Signal Averaging. J Comput Assist Tomogr 22:324-333.

Hong S-J, Bernhardt BC, Caldairou B, Hall JA, Guiot MC, Schrader D, Bernasconi N, Bernasconi A. 2017. Multimodal MRI profiling of focal cortical dysplasia type II. Neurology 88:734-742. doi:10.1212/WNL.0000000000003632

Hong S-J, Bernhardt BC, Schrader DS, Bernasconi N, Bernasconi A. 2016. Wholebrain MRI phenotyping in dysplasia-related frontal lobe epilepsy. Neurology 86:643-650. doi:10.1212/WNL.0000000000002374

Hopf A. 1968. Photometric studies on the myeloarchitecture of the human temporal lobe. J Hirnforsch 10:285-297.

Hopf A. 1957. Über die Verteilung myeloarchitektonischer Merkmale in der Scheitellappenrinde beim Menschen 26.

Hopf A. 1956. Über die Verteilung myeloarchitektonischer Merkmale in der Stirnhirnrinde beim Menschen 23.

Hopf A. 1955. Über die Verteilung myeloarchitektonischer Merkmale in der isokortikalen Schläfenlappenrinde beim Menschen 19. 
504

505

506

507

508

509

510

511

512

513

514

515

516

517

518

519

520

521

522

523

524

525

526

527

528

529

530

531

532

533

534

535

536

537

538

539

540

541

542

543

544

545

546

547

548

549

550
Huntenburg JM, Bazin P-L, Goulas A, Tardif CL, Villringer A, Margulies DS. 2017. A Systematic Relationship Between Functional Connectivity and Intracortical Myelin in the Human Cerebral Cortex. Cereb Cortex 27:981-997. doi:10.1093/cercor/bhx030

Jovicich J, Czanner S, Greve D, Haley E, Kouwe A van der, Gollub R, Kennedy D, Schmitt F, Brown G, MacFall J, Fischl B, Dale A. 2006. Reliability in multisite structural MRI studies: Effects of gradient non-linearity correction on phantom and human data. NeuroImage 30:436-443. doi:DOI: 10.1016/j.neuroimage.2005.09.046

Lazari A, Lipp I. 2021. Can MRI measure myelin? Systematic review, qualitative assessment, and meta-analysis of studies validating microstructural imaging with myelin histology. NeuroImage 230:117744. doi:10.1016/j.neuroimage.2021.117744

Lee KH, Chung K, Chung JM, Coggeshall RE. 1986. Correlation of cell body size, axon size, and signal conduction velocity for individually labelled dorsal root ganglion cells in the cat. J Comp Neurol 243:335-346. doi:10.1002/cne.902430305

Lorenz S, Weiner KS, Caspers J, Mohlberg H, Schleicher A, Bludau S, Eickhoff SB, Grill-Spector K, Zilles K, Amunts K. 2017. Two New Cytoarchitectonic Areas on the Human Mid-Fusiform Gyrus. Cereb Cortex N Y N 1991 27:373-385. doi:10.1093/cercor/bhv225

Lutti A, Dick F, Sereno MI, Weiskopf N. 2014. Using high-resolution quantitative mapping of R1 as an index of cortical myelination. NeuroImage 93 Pt 2:176188. doi:10.1016/j.neuroimage.2013.06.005

Mancini M, Karakuzu A, Cohen-Adad J, Cercignani M, Nichols TE, Stikov N. 2020. An interactive meta-analysis of MRI biomarkers of myelin. eLife 9:e61523. doi:10.7554/eLife.61523

Marques JP, Gruetter R. 2013. New developments and applications of the MP2RAGE sequence--focusing the contrast and high spatial resolution R1 mapping. PloS One 8:e69294. doi:10.1371/journal.pone.0069294

Marques JP, Khabipova D, Gruetter R. 2017. Studying cyto and myeloarchitecture of the human cortex at ultra-high field with quantitative imaging: R1, R2* and magnetic susceptibility. NeuroImage 147:152-163. doi:10.1016/j.neuroimage.2016.12.009

Mendes N, Oligschläger S, Lauckner ME, Golchert J, Huntenburg JM, Falkiewicz M, Ellamil M, Krause S, Baczkowski BM, Cozatl R, Osoianu A, Kumral D, Pool J, Golz L, Dreyer M, Haueis P, Jost R, Kramarenko Y, Engen H, Ohrnberger K, Gorgolewski KJ, Farrugia N, Babayan A, Reiter A, Schaare HL, Reinelt J, Röbbig J, Uhlig M, Erbey M, Gaebler M, Smallwood J, Villringer A, Margulies DS. 2019. A functional connectome phenotyping dataset including cognitive state and personality measures. Sci Data 6:180307. doi:10.1038/sdata.2018.307

Micheva KD, Wolman D, Mensh BD, Pax E, Buchanan J, Smith SJ, Bock DD. 2016. A large fraction of neocortical myelin ensheathes axons of local inhibitory neurons. eLife 5:e15784. doi:10.7554/eLife.15784

Nieuwenhuys R. 2013. The myeloarchitectonic studies on the human cerebral cortex of the Vogt-Vogt school, and their significance for the interpretation of 
functional neuroimaging data. Brain Struct Funct 218:303-352. doi:10.1007/s00429-012-0460-z

Nieuwenhuys R, Broere CAJ. 2020. A detailed comparison of the cytoarchitectonic and myeloarchitectonic maps of the human neocortex produced by the VogtVogt school. Brain Struct Funct. doi:10.1007/s00429-020-02150-2

Nieuwenhuys R, Broere CAJ. 2017. A map of the human neocortex showing the estimated overall myelin content of the individual architectonic areas based on the studies of Adolf Hopf. Brain Struct Funct 222:465-480. doi:10.1007/s00429-016-1228-7

Nieuwenhuys R, Broere CAJ, Cerliani L. 2015. A new myeloarchitectonic map of the human neocortex based on data from the Vogt-Vogt school. Brain Struct Funct 220:2551-2573. doi:10.1007/s00429-014-0806-9

Nord CL, Kim S-G, Callesen MB, Kvamme TL, Jensen M, Pedersen MU, Thomsen KR, Voon V. 2019. The myeloarchitecture of impulsivity: premature responding in youth is associated with decreased myelination of ventral putamen. Neuropsychopharmacol Off Publ Am Coll Neuropsychopharmacol 44:1216-1223. doi:10.1038/s41386-019-0343-6

O'Brien KR, Kober T, Hagmann P, Maeder P, Marques J, Lazeyras F, Krueger G, Roche A. 2014. Robust T1-Weighted Structural Brain Imaging and Morphometry at 7T Using MP2RAGE. PLoS ONE 9. doi:10.1371/journal.pone.0099676

O’Muircheartaigh J, Vavasour I, Ljungberg E, Li DKB, Rauscher A, Levesque V, Garren H, Clayton D, Tam R, Traboulsee A, Kolind S. 2019. Quantitative neuroimaging measures of myelin in the healthy brain and in multiple sclerosis. Hum Brain Mapp 40:2104-2116. doi:10.1002/hbm.24510

Pijnenburg R, Scholtens LH, Ardesch DJ, de Lange SC, Wei Y, van den Heuvel MP. 2021. Myelo- and cytoarchitectonic microstructural and functional human cortical atlases reconstructed in common MRI space. NeuroImage 239:118274. doi:10.1016/j.neuroimage.2021.118274

Rahmanzadeh R, Lu P-J, Barakovic M, Weigel M, Maggi P, Nguyen TD, Schiavi S, Daducci A, La Rosa F, Schaedelin S, Absinta M, Reich DS, Sati P, Wang Y, Cuadra MB, Radue E-W, Kuhle J, Kappos L, Granziera C. 2021. Myelin and axon pathology in multiple sclerosis assessed by myelin water and multi-shell diffusion imaging. Brain. doi:10.1093/brain/awab088

Royer J, Paquola C, Larivière S, Vos de Wael R, Tavakol S, Lowe AJ, Benkarim O, Evans AC, Bzdok D, Smallwood J, Frauscher B, Bernhardt BC. 2020. Myeloarchitecture gradients in the human insula: Histological underpinnings and association to intrinsic functional connectivity. NeuroImage 216:116859. doi:10.1016/j.neuroimage.2020.116859

Scholtens LH, de Reus MA, de Lange SC, Schmidt R, van den Heuvel MP. 2018. An MRI Von Economo - Koskinas atlas. NeuroImage, Segmenting the Brain 170:249-256. doi:10.1016/j.neuroimage.2016.12.069

Scholtens LH, de Reus MA, van den Heuvel MP. 2015. Linking contemporary high resolution magnetic resonance imaging to the von economo legacy: A study on the comparison of MRI cortical thickness and histological measurements of cortical structure. Hum Brain Mapp 36:3038-3046. doi:10.1002/hbm. 22826 
Sengupta S, Fritz FJ, Harms RL, Hildebrand S, Tse DHY, Poser BA, Goebel R, Roebroeck A. 2018. High resolution anatomical and quantitative MRI of the entire human occipital lobe ex vivo at 9.4T. NeuroImage, Neuroimaging with Ultra-high Field MRI: Present and Future 168:162-171. doi:10.1016/j.neuroimage.2017.03.039

Smith GE. 1927. Die Cytoarchitektonik der Hirnrinde des erwachsenen Menschen. $J$ Anat 61:264-266.

Sprooten E, O'Halloran R, Dinse J, Lee WH, Moser DA, Doucet GE, Goodman M, Krinsky H, Paulino A, Rasgon A, Leibu E, Balchandani P, Inglese M, Frangou S. 2019. Depth-dependent intracortical myelin organization in the living human brain determined by in vivo ultra-high field magnetic resonance imaging. NeuroImage 185:27-34. doi:10.1016/j.neuroimage.2018.10.023

Strasburger EH. 1937. Die myeloarchitektonische Gliederung des Stirnhirns beim Menschen und Schimpansen 42.

Stüber C, Morawski M, Schäfer A, Labadie C, Wähnert M, Leuze C, Streicher M, Barapatre N, Reimann K, Geyer S, Spemann D, Turner R. 2014. Myelin and iron concentration in the human brain: a quantitative study of MRI contrast. NeuroImage 93 Pt 1:95-106. doi:10.1016/j.neuroimage.2014.02.026

Talairach J, Tournoux P, Missir O. 1993. Referentially Oriented Cerebral MRI Anatomy: An Atlas of Stereotaxic Anatomical Correlations for Gray and White Matter. G. Thieme Verlag.

Tomassy GS, Berger DR, Chen H-H, Kasthuri N, Hayworth KJ, Vercelli A, Seung HS, Lichtman JW, Arlotta P. 2014. Distinct profiles of myelin distribution along single axons of pyramidal neurons in the neocortex. Science 344:319324. doi:10.1126/science. 1249766

Trampel R, Bazin P-L, Pine K, Weiskopf N. 2019. In-vivo magnetic resonance imaging (MRI) of laminae in the human cortex. NeuroImage 197:707-715. doi:10.1016/j.neuroimage.2017.09.037

Triarhou LC. 2007. Constantin von Economo (1876-1931). J Neurol 254:550-551. doi:10.1007/s00415-006-0308-7

van der Weijden CWJ, García DV, Borra RJH, Thurner P, Meilof JF, van Laar P-J, Dierckx PRAJO, Gutmann IW, de Vries EFJ. 2020. Myelin quantification with MRI: A systematic review of accuracy and reproducibility. NeuroImage 117561. doi:10.1016/j.neuroimage.2020.117561

Vogt C, Vogt O. 1919. Allgemeine Ergebnisse unserer Hirnforschung.

Waehnert MD, Dinse J, Schäfer A, Geyer S, Bazin P-L, Turner R, Tardif CL. 2016. A subject-specific framework for in vivo myeloarchitectonic analysis using high resolution quantitative MRI. NeuroImage 125:94-107. doi:10.1016/j.neuroimage.2015.10.001

Waehnert MD, Dinse J, Weiss M, Streicher MN, Waehnert P, Geyer S, Turner R, Bazin P-L. 2014. Anatomically motivated modeling of cortical laminae. NeuroImage, In-vivo Brodmann Mapping of the Human Brain 93:210-220. doi:10.1016/j.neuroimage.2013.03.078

Weiskopf N, Mohammadi S, Lutti A, Callaghan MF. 2015. Advances in MRI-based computational neuroanatomy: from morphometry to in-vivo histology. Curr Opin Neurol 28:313-322. doi:10.1097/WCO.0000000000000222 
643 Yuan D, Luo D, Kwok VPY, Zhou Y, Tian H, Yu Q, An J, Gao J-H, Qiu S, Tan LH. 644 2021. Myeloarchitectonic Asymmetries of Language Regions in the Human $645 \quad$ Brain. Cereb Cortex N Y N 1991 31:4169-4179. doi:10.1093/cercor/bhab076

646 Zhang H, Schneider T, Wheeler-Kingshott CA, Alexander DC. 2012. NODDI:

650 Zilles K, Amunts K. 2010. Centenary of Brodmann's map--conception and fate. Nat 651 Rev Neurosci 11:139-145. doi:10.1038/nrn2776

652 Zilles K, Palomero-Gallagher N, Amunts K. 2015. Myeloarchitecture and Maps of the 653 Cerebral Cortex In: Toga AW, editor. Brain Mapping. Waltham: Academic Press. pp. 137-156. doi:10.1016/B978-0-12-397025-1.00209-8

655 
bioRxiv preprint doi: https://doi.org/10.1101/2022.01.17.476369; this version posted January 20, 2022. The copyright holder for this preprint (which was not certified by peer review) is the author/funder. All rights reserved. No reuse allowed without permission.

\section{Figures}

\section{8}

\section{Figures 1}
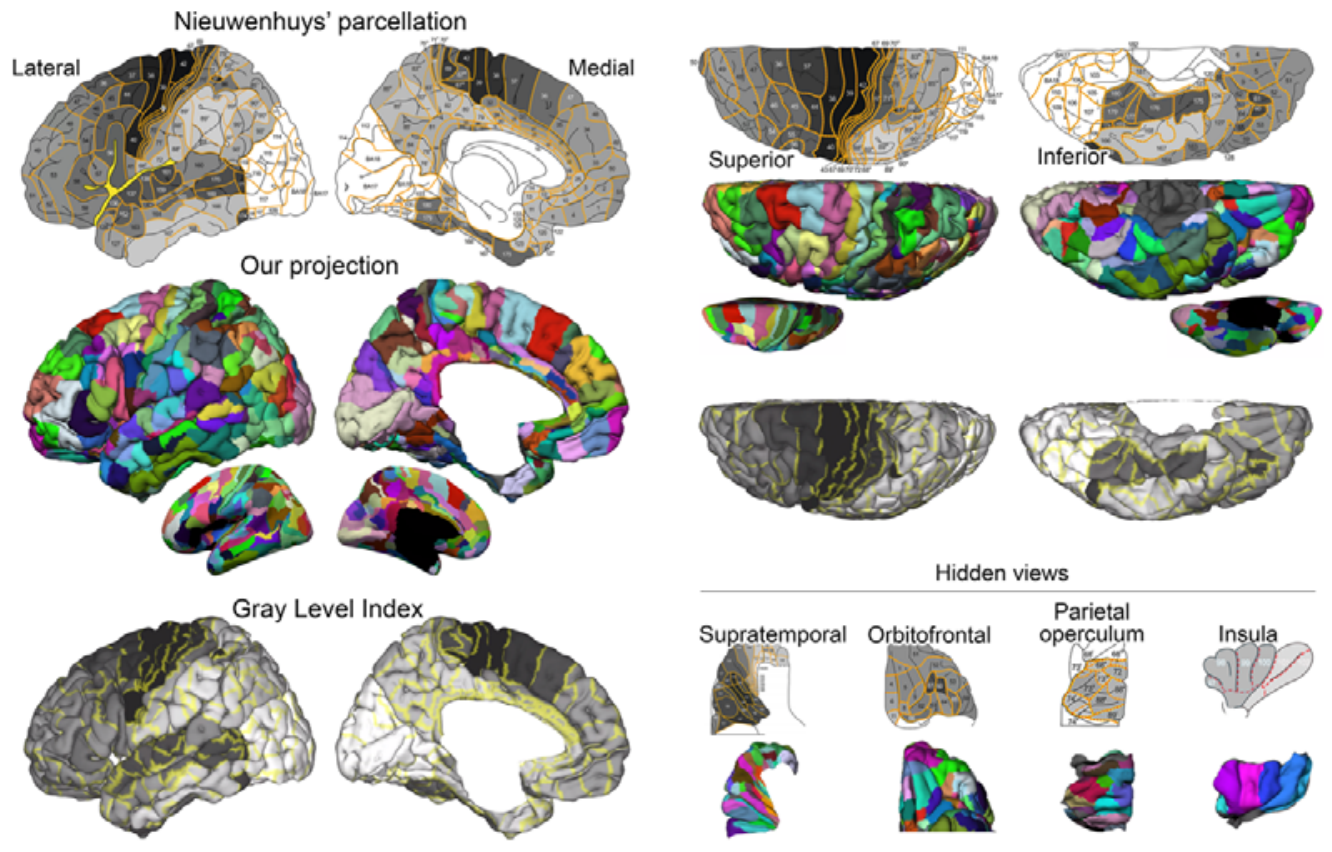

FIGURE 1. Construction of the 3D myeloarchitectonic MNI atlas (MYATLAS). Manual cortical parcellations (based on the topological transformation of Vogt-Vogt parcellation) across view planes (lateral, medial, superior, inferior; on pial and inflated surfaces), with the 3D projection and mean gray level index measuring the degrees of myelination. Hidden areas within the orbitofrontal region, the supratemporal lobe, and the parietal operculum were labeled by extracting view planes similar to the original publication; they were then merged back with the whole-brain surface once segmentations were finished (panel on the right bottom). 
bioRxiv preprint doi: https://doi.org/10.1101/2022.01.17.476369; this version posted January 20, 2022. The copyright holder for this preprint (which was not certified by peer review) is the author/funder. All rights reserved. No reuse allowed without permission.

Figure 2

A. Myeloarchitectonic categories

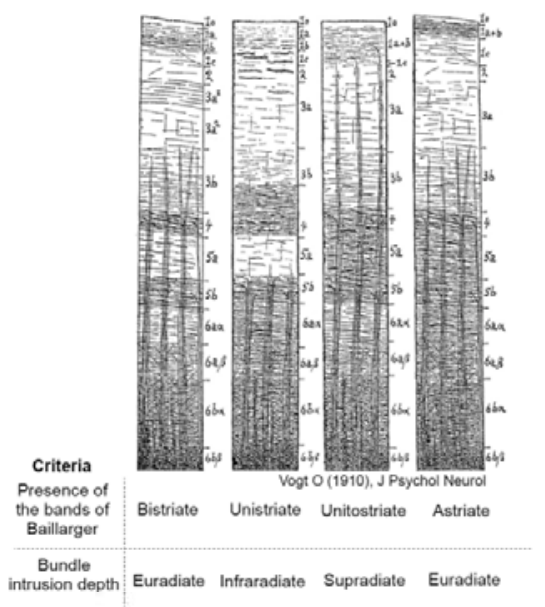

C. Intracortical depth profiles
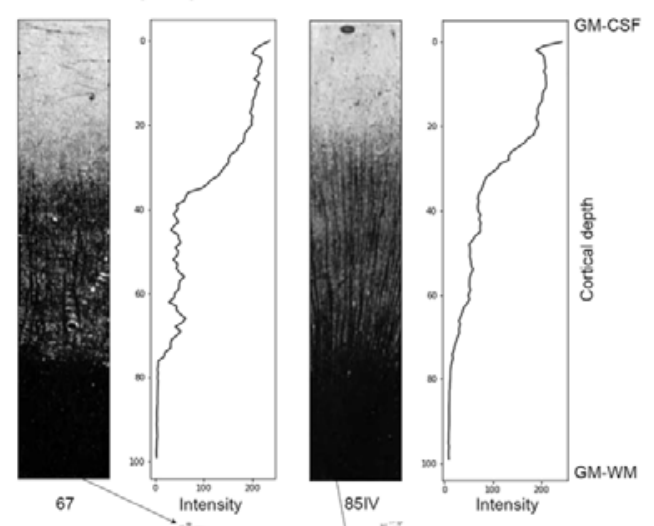

B. Myeloarchitectural category maps

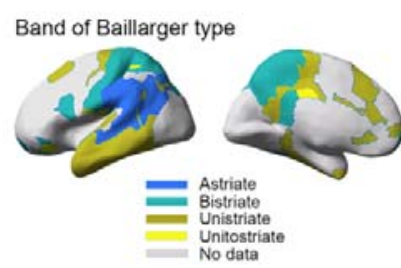

Bundle intrusion type

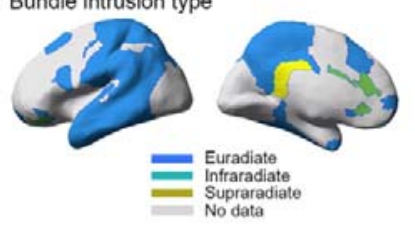

670

671

672

673

674

675

676

Figure 2. Intracortical depth profiling based on Vogt-Hopf histological data. A) Categories of myeloarchitectonic features based on laminar and bundle intrusion patterns; B) Mapping of feature information onto the cortical surface; no data indicate subtype information unavailable from the original literature. C) Examples of myelin-stained cortical areas across full cortical depth with their corresponding depth profiles (maps shown in Supplementary Material 1). 
bioRxiv preprint doi: https://doi.org/10.1101/2022.01.17.476369; this version posted January 20, 2022. The copyright holder for this preprint (which was not certified by peer review) is the author/funder. All rights reserved. No reuse allowed without permission.

\section{$678 \quad$ Figure 3}

A. Correlation between MGL and T1q MRI
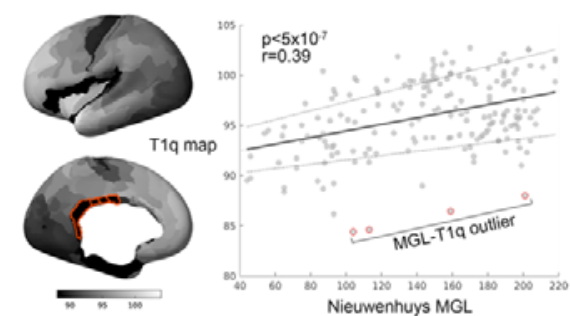
Nieuwenhuys MGL

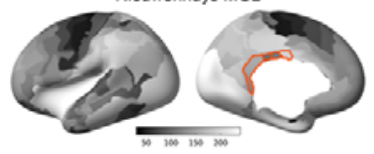

B. Correlation between histological and MRI depth profiles

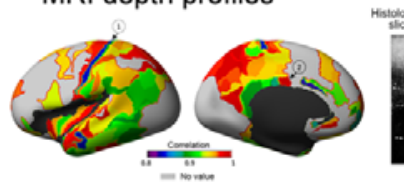

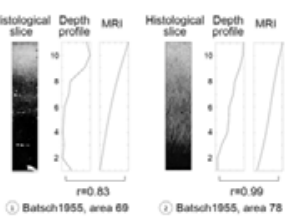

C. Correlation between MGL and cytoarchitectures
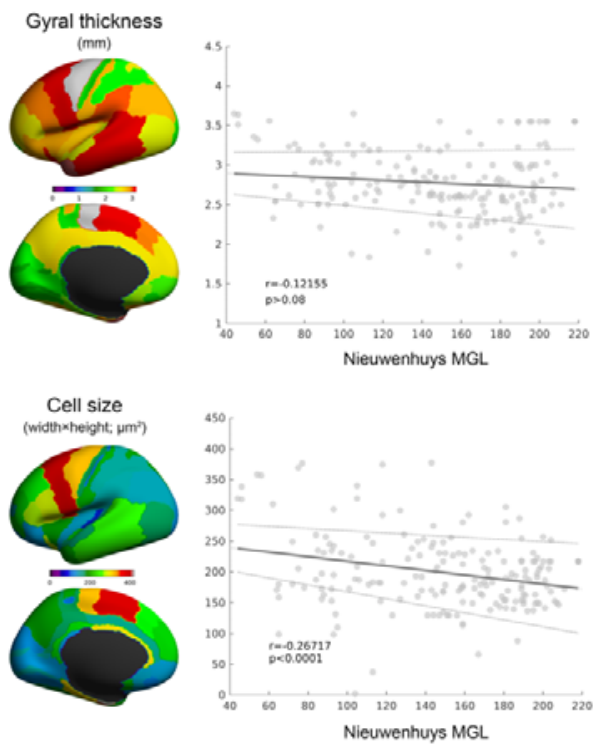

680 Figure 3. Correlation of in vivo MRI markers and cytoarchitectural features. A) Whole-brain spatial correlation between in vivo quantitative T1w MRI (T1q) and mean gray level (MGL) index. Outliers ( $> \pm 2 \mathrm{SD} q \mathrm{qT} 1)$ in the posterior cingulate are in red). B) Whole-brain depth profile correlation between qT1 and photodensitometry-derived cortical myelin content; only areas with available depth profiles are presented. Two areas with the highest and lowest correlation between histology and qT1 MRI are given as examples. C) Whole-brain spatial correlation between MGL and cytoarchitectural 687 features derived from von Economo-Koskinas literature (i.e., gyral dome thickness and cell size). 
bioRxiv preprint doi: https://doi.org/10.1101/2022.01.17.476369; this version posted January 20, 2022. The copyright holder for this preprint (which was not certified by peer review) is the author/funder. All rights reserved. No reuse allowed without permission.

689 Supplementary Table 1 - Label indices for discordant views across brain areas

\begin{tabular}{ccc} 
Area index & $\begin{array}{c}\text { Discordant views } \\
\text { (See Figure 1C) }\end{array}$ & View followed \\
\hline 109 & Lateral, inferior & inferior \\
111 & Lateral, medial & Split into two segments \\
112 & Lateral, medial & Split into two segments \\
120 & Lateral, medial & medial \\
127 & Lateral, inferior & lateral \\
128 & Lateral, inferior & Lateral \\
175 & Medial, inferior & inferior \\
176 & Medial, inferior & inferior \\
178 & Medial, inferior & inferior
\end{tabular}


bioRxiv preprint doi: https://doi.org/10.1101/2022.01.17.476369; this version posted January 20, 2022. The copyright holder for this preprint (which was not certified by peer review) is the author/funder. All rights reserved. No reuse allowed without permission.

\section{Supplementary Table 2 -FreeSurfer command line code}

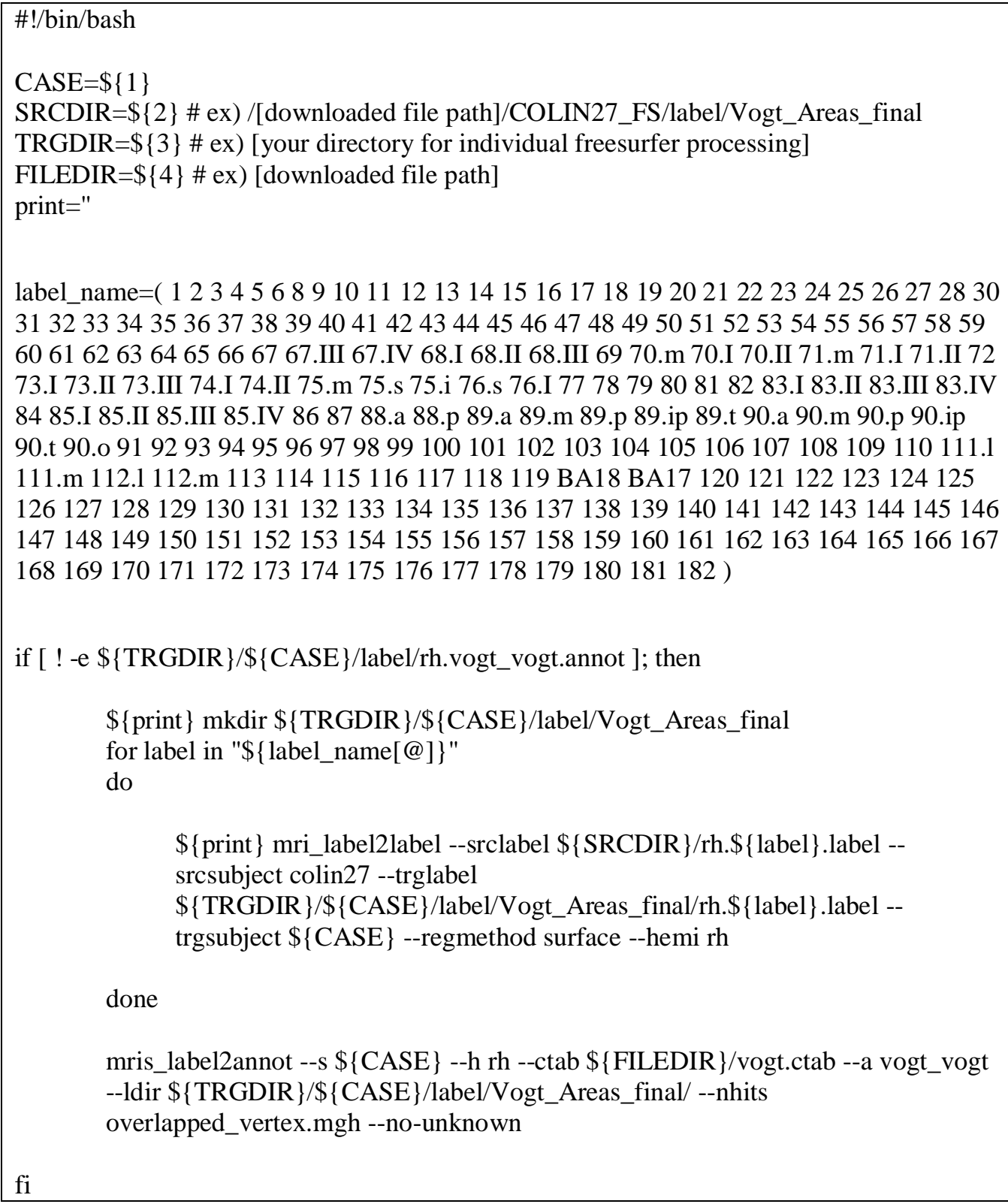

